\title{
Quasi-Homogeneous Backward-Wave Plasmonic Structures: Theory and Accurate Simulation
}

\author{
Igor Tsukerman \\ Department of Electrical and Computer Engineering, \\ The University of Akron, $\mathrm{OH}$ 44325-3904, USA*
}

\begin{abstract}
Backward waves and negative refraction are shown to exist in plasmonic crystals whose lattice cell size is a very small fraction of the vacuum wavelength (less than $1 / 40$ th in an illustrative example). Such "quasi-homogeneity" is important, in particular, for high-resolution imaging. Real and complex Bloch bands are computed using the recently developed finite-difference calculus of "Flexible Local Approximation MEthods" (FLAME) that produces linear eigenproblems, as opposed to quadratic or nonlinear ones typical for other techniques. FLAME dramatically improves the accuracy by incorporating local analytical approximations of the solution into the numerical scheme.
\end{abstract}

Backward waves (Poynting vector opposite to phase velocity [29]) and the closely related phenomenon of negative refraction have been extensively studied in recent years (e.g. $[9,12]$ and references there) due to the intriguing physical effects and potential applications in imaging and other areas.

Backward waves in periodic structures may in general exist only if the lattice cell size, as a fraction of the vacuum wavelength $\lambda_{0}$, is above certain thresholds derived recently in [23]. Plasmonic crystals [4, 14] are an interesting exception because in the vicinity of a plasmon resonance the constraints on the cell size are relaxed or removed, as explained below, and thus it may be possible to reduce the lattice cell size and approach an ideal homogeneous negative-index medium.

Bloch modes play a central role in the analysis of electromagnetic waves in periodic structures. While analytical expressions for these modes are available only in special one-dimensional cases $[11,22,26]$, there exist a variety of computational techniques: Fourier transforms (plane wave expansion, PWE) [7, 11, 22], scattering matrices and lattice summation [2], finite difference [25, 27] and finite element $[1,4,5,8,17]$ analysis, semi-analytical methods [28], and more.

Bloch wave problems have three (in 2D) or four (in 3D) scalar eigenparameters: frequency $\omega$ and the Cartesian components of the Bloch vector $\mathbf{K}$. Solving for all these parameters, and the respective eigenmodes, simultaneously is impractical. The usual approach is to look for the values of $\omega$ for any given $\mathbf{K}$. The differential operator of the problem, and hence the respective matrices in the numerical computation, contain the permittivity $\epsilon$ and therefore for dispersive media $(\epsilon=\epsilon(\omega))$ depend on the frequency in a complicated way. Consequently, the resulting eigenvalue problems with respect to $\omega$ are nonlinear. Several solution methods have been proposed $[16,19]$ but are not simple, and convergence is not guaranteed.

A more elegant approach, where frequency is treated

*Electronic address: igor@uakron.edu as a given parameter and components of the Bloch vector as unknown eigenvalues, has been explored relatively recently in PWE [13] and in FEM [4, 17]. Quadratic eigenproblems with respect to the Bloch number usually arise and can be converted to linear ones by introducing auxiliary unknowns either on the continuous level (e.g. solving for both fields $\mathbf{E}, \mathbf{H}$ instead of just one) or, alternatively, on the linear algebra level [18]. This conversion doubles the number of unknowns; the computational cost, typically proportional to the cube of the system size, increases about eightfold.

In the recently developed generalized finite-difference (FD) calculus of Flexible Local Approximation MEthods (FLAME [21, 22, 24]) high accuracy is achieved by replacing the Taylor expansions of standard FD analysis with much better approximating functions, e.g. plane waves or cylindrical harmonics. In FLAME, $\omega$ is a natural "independent variable" because the approximating functions are derived for a fixed value of $\omega$. FLAME has two clear advantages in the computation of (real or complex) Bloch modes: (i) it dramatically improves the accuracy by incorporating local analytical approximations of the solution into the numerical scheme (see examples below); (ii) it produces linear eigenproblems.

Let us consider band structure calculation in a photonic crystal formed by an infinite lattice of rectangular cells $L_{x} \times L_{y}$ in the $x y$-plane. In a very common case, each cell contains a dielectric cylindrical rod with a radius $r_{\text {rod }}$ and the relative dielectric permittivity $\epsilon_{\text {rod }}$. The medium outside the rod has permittivity $\epsilon_{\text {out }}$. At optical frequencies, all media are intrinsically nonmagnetic. The governing wave equation for the TE mode (one-component magnetic field phasor $H=H_{z}$ ) is

$$
\nabla \cdot \epsilon^{-1} \nabla H+\omega^{2} \mu_{0} H=0
$$

where the relative dielectric permittivity $\epsilon=\epsilon(x, y)$ is periodic over the lattice. The $\exp (-\mathrm{i} \omega t)$ convention is used for complex phasors. The $H$ field is sought as a Bloch-Floquet wave [11] with a (yet undetermined) Bloch vector $\mathbf{K}_{B}$ :

$$
H(\mathbf{r})=H_{\mathrm{PER}}(\mathbf{r}) \exp \left(\mathrm{iK}_{B} \cdot \mathbf{r}\right) \quad \mathbf{r} \equiv(x, y)
$$

where $H_{\mathrm{PER}}$ is periodic over the lattice. In the space of Bloch vectors $\mathbf{K}_{B}$, the first Brillouin zone is 

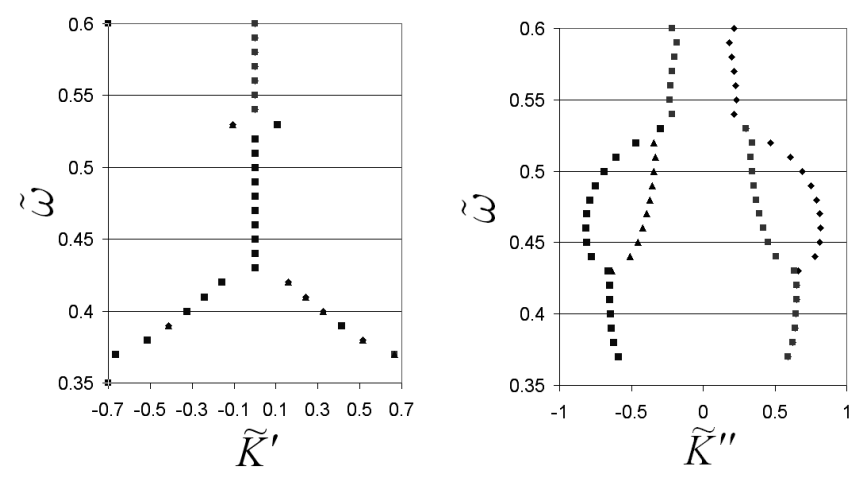

FIG. 1: A fragment of the band diagram of the Davanco et al. [4] plasmonic crystal. $\tilde{\omega}=\omega a / c, \tilde{K}=K_{B} a / \pi$.

$\left[-\pi / L_{x}, \pi / L_{x}\right] \times\left[-\pi / L_{y}, \pi / L_{y}\right]$. For notational simplicity and without real loss of generality, let $L_{x}=L_{y}=a$.

There are two general options: solving for the periodic factor $H_{\mathrm{PER}}(x, y)$ or, alternatively, for the full $H$-field of (1). In the first case, standard periodic boundary conditions apply, but the differential operator is more complicated than in the second case. The boundary conditions for the full $H$-field are "scaled-periodic" due to the Bloch exponential $\exp (\mathrm{i} \mathbf{K} \cdot \mathbf{r})$ :

$H(a / 2, y)=\exp \left(\mathrm{i} K_{x} a\right) H(-a / 2, y) ; \quad|y| \leq a / 2$ with similar conditions at the boundaries $y= \pm a / 2$.

Accurate local analytical approximations that FLAME relies on are available for the full $H$-field formulation and involve Bessel / Hankel functions [20, 21, 22]. Two Bloch conditions - one for the $H$ field and another one for the $E$ field - need to be imposed on the cell boundaries; the implementation details are described in [24]. In matrix-vector form, the FLAME eigenvalue problem is $L \underline{E}=\left(b_{x} B_{x}+b_{y} B_{y}\right) \underline{E}$, where $\underline{E}$ is the Euclidean vector of the nodal values of the field on the grid, and $b_{x}, b_{y}$ are the Bloch factors $b_{x}=\exp \left(\mathrm{i} K_{x} L_{x}\right) ; b_{y}=\exp \left(\mathrm{i} K_{y} L_{y}\right)$. Matrix $L$ has a sparse structure typical of finite-difference methods; matrix $B$ is extremely sparse - its nonzero entries correspond only to two layers of grid nodes adjacent to the cell boundary.

Numerical examples for real-K modes in regular dielectrics are given in [24]. To illustrate the computation of complex modes in plasmonic crystals, let us start, for convenience of comparison, with the same example as in [4]. The permittivity of the rods is assumed to be described by the normalized Drude model $\epsilon(\tilde{\omega})=1-\tilde{\omega}^{-1}\left(\tilde{\omega}-\mathrm{i} \tilde{\omega}_{c}\right)^{-1}$ with the normalized frequencies $\tilde{\omega}=\omega / \omega_{p}, \tilde{\omega}_{c}=\omega_{c} / \omega_{p}$, where $\omega_{p}$ is the plasma frequency, $\omega_{c}$ is the damping rate, and $c$ is the speed of light in free space. The square lattice cell size is $a=c / \omega_{p}$ and the cylinder radii are $r_{\text {rod }}=0.45 a$. The Bloch modes computed with the 9-point FLAME scheme coincide with the ones reported in [4]; a fragment of the band diagram computed with FLAME for $\omega_{c}=0$ is shown in Fig. 1 for reference. The accuracy of FLAME is remarkably high: as evidenced by the typical convergence results for several modes in Table I, on the $40 \times 40$ grid the FLAME

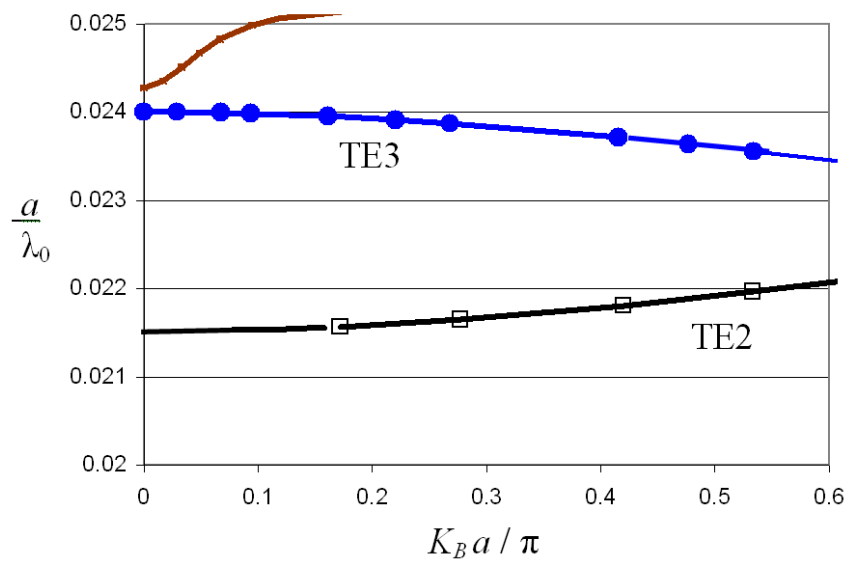

FIG. 2: A backward-wave mode (TE3, circles, dotted line) in a plasmonic crystal with the lattice cell size as small as $\sim \lambda_{0} / 40$. Parameters: $\tilde{\omega}_{p}=0.25, r_{\text {rod }}=0.45 a$.

\begin{tabular}{|c|c|c|c|}
\hline Grid & $K_{\text {real }}$ & $K_{1}^{\prime \prime}$ & $K_{2}^{\prime \prime}$ \\
\hline $20 \times 20$ & 0.241048852 & 2.573701526 & 4.113530131 \\
\hline $30 \times 30$ & 0.240708525 & 2.572916824 & 4.108636747 \\
\hline $40 \times 40$ & 0.240652136 & 2.572740104 & 4.544368769 \\
\hline $50 \times 50$ & 0.240644493 & 2.572711175 & 4.544040773 \\
\hline
\end{tabular}

TABLE I: Typical convergence of the numerical values for Bloch wavenumbers. Results for $\tilde{\omega}=0.26$. $K_{\text {real }}$ is a real mode. Modes $K_{1,2}^{\prime \prime}$ have the real part $K^{\prime}=\pi / a$.

results already have about five correct digits - several orders of magnitude higher accuracy than in a typical PWE calculation [11, 22, 24]. Note that this example is not computationally favorable because the filling factor is high and the gaps between the rods are narrow.

There is some similarity between FLAME and the semi-analytical construction of [28], where the field in the whole lattice cell is expanded into cylindrical harmonics and an eigenvalue problem for the coefficients of this expansion is obtained by imposing the Bloch boundary conditions at a set of collocation points on the cell boundary. In contrast, FLAME uses only local analytical approximations that are valid over each individual grid stencil. Therefore FLAME can be extended to more general situations, e.g. with more than one rod in the cell, non-circular shapes, defects, photonic waveguides, etc. $[3,10,22]$, where global analytical solutions are not available.

Backward waves. As shown in [23], the cell size of periodic dielectric structures capable of supporting backward waves must lie above a fundamental threshold:

$$
|\eta| \leq\left(4 \pi^{2}\right)^{-1}|\epsilon|_{\max }\left(1+|\lambda|_{\max }\left(\mathcal{L}_{\epsilon}^{-1}\right)|\epsilon|_{\max }\right)
$$

where $\eta=\tilde{\omega}^{-2} ; \tilde{\omega}=\omega a / c=2 \pi a / \lambda_{0} ;|\lambda|_{\max }\left(\mathcal{L}_{\epsilon}^{-1}\right)$ is the maximum eigenvalue of the inverse of the electrostatic operator $\mathcal{L}_{\epsilon}=\tilde{\nabla} \cdot \epsilon \tilde{\nabla}$. This eigenvalue is bounded unless the operating frequency is close to the quasistatic plasmon resonance value. In particular, for non- 


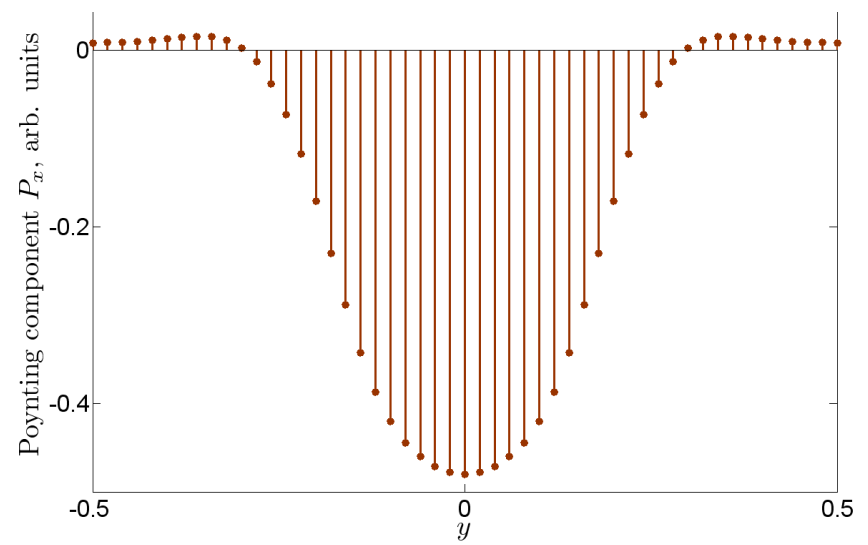

FIG. 3: Predominantly negative power density on the right edge of the very small lattice cell $\left(a / \lambda_{0}=0.02387\right)$.

plasmonic materials with $0<\epsilon_{\min } \leq \epsilon \leq \epsilon_{\max }$ throughout the lattice cell, the lower cell size bound specializes to $\left(a / \lambda_{0}\right)^{2} \geq\left[|\epsilon|_{\max }\left(1+|\epsilon|_{\max } /\left(4 \pi^{2} \epsilon_{\min }\right)\right)\right]^{-1}$ [23]. The plasmonic case is thus exceptional, because at the plasmonic resonance the electrostatic operator in (4) becomes singular and the constraint on the cell size disappears.

The following example demonstrates that backward waves, and hence negative refraction, can be obtained in plasmonic crystals with very small lattice cells (here $\sim \lambda_{0} / 40$ ). As before, consider a square lattice of cylindrical rods, with $r_{\text {rod }}=0.45 a$. For this "proof-of-concept" example, losses are neglected and the Drude-like dielectric function is set as $\epsilon_{\text {rod }}=1-\tilde{\omega}_{p}^{2} / \tilde{\omega}^{2}$, with $\tilde{\omega}_{p}=\omega_{p} a / c$. A fragment of the band diagram in the $\Gamma X$ direction for $\tilde{\omega}_{p}=0.25$ is shown in Fig. 2. The TE3 band (circles, solid line) corresponds to a backward wave: (a) the group velocity $\partial \omega / \partial K_{B}$ for this band is negative; (b) the $x$ component of the Poynting vector, plotted in Fig. 3, clearly indicates negative energy flow; (c) the first-Brillouin-zone component of the wave is dominant, as can be confirmed by Fourier analysis of the periodic field $H_{\mathrm{PER}}(x, y)$ in (2); hence the wave has a well defined positive phase velocity.

In summary, negative refraction can be obtained in a quasi-homogeneous medium with a very small cell size as a "plasmonic exception" circumventing the theoretical cell size bounds of [23]. This may have important implications for imaging, as the inhomogeneity of the medium limits the resolution [15, 23]. For illustration, a backward-wave mode is demonstrated in a plasmonic crystal with the cell size of less than $1 / 40$ th of the vacuum wavelength. FLAME, a generalized finite-difference calculus based on very accurate local approximations of the solution, was applied to the computation of real and complex Bloch bands.
[1] W. Axmann and P. Kuchment. An efficient finite element method for computing spectra of photonic and acoustic band-gap materials - I. Scalar case. J. of Comp. Phys., 150(2):468-481, 1999.

[2] L. C. Botten et al. in Electromagnetic Theory and Applications for Photonic Crystals. CRC Press, 2005.

[3] J. Dai and I. Tsukerman. Flexible approximation schemes with adaptive grid refinement. IEEE Trans. Magn., 44(6):1206-1209, 2008.

[4] M. Davanco, Y. Urzhumov, and G. Shvets. The complex Bloch bands of a $2 \mathrm{D}$ plasmonic crystal displaying isotropic negative refraction. Opt Exp, 15:9681-9691, 2007.

[5] D.C. Dobson and J.E. Pasciak. Analysis of an algorithm for computing electromagnetic Bloch modes using Nedelec spaces. Comput. Meth. in Appl. Math., 1(2):138-153, 2001.

[6] A.J. Hoffman et al. Negative refraction in semiconductor metamaterials. Nature Mat, 6:946-950, 2007.

[7] S. G. Johnson and J.D. Joannopoulos. Block-iterative frequency-domain methods for maxwell's equations in a planewave basis. Opt. Exp, 8(3):173-190, 2001.

[8] C. Mias, J. P. Webb, and R. L. Ferrari. Finite element modeling of electromagnetic waves in doubly and triply periodic structures. IEE Proc. Optoel, 146:111-118, 1999.

[9] J.B. Pendry and D.R. Smith. Reversing light with negative refraction. Phys. Today, 57:37-43, 2004.

[10] H. Pinheiro, J.P. Webb, and I. Tsukerman. Flexible local approximation models for wave scattering in photonic crystal devices. IEEE Trans. Magn., 43(4):1321-1324,
2007.

[11] K. Sakoda. Optical Properties of Photonic Crystals. Berlin; New York: Springer, 2005.

[12] V. M. Shalaev. Optical negative-index metamaterials. Nat Photonics, 1:41-48, 2006.

[13] S. Shi, C. Chen, and D. W. Prather. Revised plane wave method for dispersive material and its application to band structure calculations of photonic crystal slabs. Appl Phys Lett, 86(4):043104, 2005.

[14] G. Shvets, Y.A. Urzhumov. Engineering the electromagnetic properties of periodic nanostructures using electrostatic resonances. Phys. Rev. Lett., 93(24):243902, 2004.

[15] D. R. Smith et al. Limitations on subdiffraction imaging with a negative refractive index slab. $A P L, 82(10): 1506-$ 1508, 2003.

[16] A. Spence and C. Poulton. Photonic band structure calculations using nonlinear eigenvalue techniques. $J$. Comp. Phys., 204:65-81, 2005.

[17] A. A. Tavallaee and J. P. Webb. Finite element modeling of evanescent modes in the stopband of periodic structures. IEEE Trans. Magn., 44(6):1358-1361, 2008.

[18] F. Tisseur and K. Meerbergen. The quadratic eigenvalue problem. SIAM Rev., 43(2), 2001.

[19] O. Toader and S. John. Photonic band gap enhancement in frequency-dependent dielectrics. Phys. Rev. E, 70(4):046605, 2004.

[20] I. Tsukerman. Electromagnetic applications of a new finite-difference calculus. IEEE Trans. Magn., 41(7):2206-2225, 2005.

[21] I. Tsukerman. A class of difference schemes with flexi- 
ble local approximation. J Comp Phys, 211(2):659-699, 2006.

[22] I. Tsukerman. Computational Methods for Nanoscale Applications: Particles, Plasmons and Waves. Springer, 2007.

[23] I. Tsukerman. Negative refraction and the minimum lattice cell size. JOSA B, 25(6):927-936, 2008.

[24] I. Tsukerman and F. Cajko. Photonic band structure computation using FLAME. IEEE Trans. Magn., 44(6):1382-1385, 2008.

[25] H.Y.D. Yang. Finite difference analysis of 2-D photonic crystals. IEEE Trans MTT, 44(12):2688-2695, 1996.
[26] P. Yeh. Optical Waves in Layered Media. Hoboken, N.J.: John Wiley, 2005.

[27] C.-P. Yu and H.-C. Chang. Compact finite-difference frequency-domain method for the analysis of twodimensional photonic crystals. Opt. Exp, 12(7):13971408, 2004.

[28] J. Yuan and Y. Lu. Photonic bandgap calculations with Dirichlet-to-Neumann maps. JOSA A, 23(12):3217-3222, 2006.

[29] More generally, at an obtuse angle; but cases of strong anisotropy [6] are beyond the scope of this paper. 